\title{
Global nonexistence of solutions for a quasilinear wave equation with acoustic boundary conditions
}

\author{
Jin-Mun Jeong ${ }^{1}$, Jong-Yeoul Park² and Yong Han Kang ${ }^{3 *}$ (D)
}

"Correspondence:
yonghann@cu.ac.kr
${ }^{3}$ Institute of Liberal Education,
Catholic University of Daegu,
Gyeongsan, 712-702, South Korea
Full list of author information is
available at the end of the article

available at the end of the article

\begin{abstract}
We consider the quasilinear wave equation

$$
u_{t t}-\Delta u_{t}-\operatorname{div}\left(|\nabla u|^{\alpha-2} \nabla u\right)-\operatorname{div}\left(\left|\nabla u_{t}\right|^{\beta-2} \nabla u_{t}\right)+a\left|u_{t}\right|^{m-2} u_{t}=b|u|^{p-2} u
$$

$a, b>0$, associated with initial and Dirichlet boundary conditions at one part and acoustic boundary conditions at another part, respectively. We prove, under suitable conditions on $\alpha, \beta, m, p$ and for negative initial energy, a global nonexistence of solutions.
\end{abstract}

MSC: $35 B 40 ; 35 B 44 ; 35 L 72$

Keywords: quasilinear wave equation; blow-up; acoustic boundary

\section{Introduction}

In this paper, we consider the following quasilinear wave equation with acoustic boundary conditions:

$$
\begin{aligned}
& u_{t t}-\Delta u_{t}-\operatorname{div}\left(|\nabla u|^{\alpha-2} \nabla u\right)-\operatorname{div}\left(\left|\nabla u_{t}\right|^{\beta-2} \nabla u_{t}\right) \\
& \quad+a\left|u_{t}\right|^{m-2} u_{t}=b|u|^{p-2} u \quad \text { in } \Omega \times(0, \infty), \\
& u=0 \quad \text { on } \Gamma_{0} \times(0, \infty), \\
& \frac{\partial u_{t}}{\partial v}+|\nabla u|^{\alpha-2} \frac{\partial u}{\partial v}+\left|\nabla u_{t}\right|^{\beta-2} \frac{\partial u_{t}}{\partial v}=h(x) y_{t} \quad \text { on } \Gamma_{1} \times(0, \infty), \\
& u_{t}+f(x) y_{t}+q(x) y=0 \quad \text { on } \Gamma_{1} \times(0, \infty), \\
& u(x, 0)=u_{0}(x), \quad u_{t}(x, 0)=u_{1}(x) \quad \text { in } \Omega, \\
& y(x, 0)=y_{0}(x) \quad \text { on } \Gamma_{1} \times(0, \infty),
\end{aligned}
$$

where $a, b>0, \alpha, \beta, m, p>2, \Omega$ is a regular and bounded domain of $R^{n}(n \geq 1)$ and $\partial \Omega(=$ $\Gamma):=\Gamma_{0} \cup \Gamma_{1}$. Here $\Gamma_{0}, \Gamma_{1}$ are closed and disjoint, and $\frac{\partial}{\partial v}$ denotes the unit outer normal derivative. The functions $f, q, h: \Gamma_{1} \longrightarrow R^{+}$are essentially bounded and $0<q_{0} \leq q(x)$ on $\Gamma_{1}$.

(c) The Author(s) 2017. This article is distributed under the terms of the Creative Commons Attribution 4.0 International License (http://creativecommons.org/licenses/by/4.0/), which permits unrestricted use, distribution, and reproduction in any medium, provided you give appropriate credit to the original author(s) and the source, provide a link to the Creative Commons license, and indicate if changes were made. 
The system (1.1)-(1.6) is a model of a quasilinear wave equation with acoustic boundary conditions. The acoustic boundary conditions were introduced by Morse and Ingard [1] in 1968 and developed by Beale and Rosencrans in [2], where the authors proved the global existence and regularity of the linear problem. Furthermore, Boukhatem and Benabderrahmane [3, 4] studied the existence, blow-up and decay of solutions for viscoelastic wave equations with acoustic boundary conditions. Graber and Said-Houari [5] studied the blow-up solutions for the wave equation with semilinear porous acoustic boundary conditions. Moreover, Wu [6] also considered blow-up solutions for a nonlinear wave equation with porous acoustic boundary conditions. The global nonexistence of solutions for a class of wave equations with nonlinear damping and source terms was proved by Messaoudi and Said-Houari [7-9] (see [10-13] for more details). Recently, Piskin [14] investigated the energy decay and blow-up of solutions for quasilinear hyperbolic equations with nonlinear damping and source terms (see [15-18] for more details).

Motivated by the previous works, in this paper, we study the global nonexistence of solutions for quasilinear wave equations with acoustic boundary conditions. To the best of our knowledge, there are no results of a quasilinear wave equation with acoustic boundary conditions. This work is meaningful. The outline of the paper is the following. In Section 2, we prove the main result.

\section{Blow-up results}

In order to state and prove our result, we introduce

$$
\begin{aligned}
Z= & L^{\infty}\left([0, T) ; W^{1, \alpha}(\Omega)\right) \cap W^{1, \infty}\left([0, T) ; L^{2}(\Omega)\right) \\
& \cap W^{1, \beta}\left([0, T) ; W^{1, \beta}(\Omega)\right) \cap W^{1, m}\left([0, T) ; L^{m}(\Omega)\right)
\end{aligned}
$$

for $T>0$ and the energy functional

$$
E(t)=\frac{1}{2} \int_{\Omega} u_{t}^{2} d x+\frac{1}{\alpha} \int_{\Omega}|\nabla u|^{\alpha} d x-\frac{b}{p} \int_{\Omega}|u|^{p} d x+\frac{1}{2} \int_{\Gamma_{1}} h(x) q(x) y^{2}(t) d \Gamma .
$$

Theorem 2.1 Assume that $\alpha, \beta, m, p \geq 2$ such that $\beta<\alpha$, and $\max \{m, \alpha\}<p<r_{\alpha}$, where $r_{\alpha}$ is the Sobolev critical exponent of $W^{1, \alpha}(\Omega)$. Assume further that

$$
E(0)<0 .
$$

Then the solution $(u, y) \in Z \times L^{2}\left(R^{+} ; L^{2}\left(\Gamma_{1}\right)\right)$ of (1.1)-(1.6) can not exist for all time.

Remark 2.2 If the solution $u$ of (1.1)-(1.6) is smooth enough, then it blows up in finite time.

Proof We suppose that the solution exists for all time, and we reach a contradiction. For this purpose, we multiply Eq. (1.1) by $u_{t}$ and, using (1.2)-(1.4), we obtain

$$
\begin{aligned}
E^{\prime}(t)= & -\int_{\Omega}\left|\nabla u_{t}(t)\right|^{2} d x-\int_{\Omega}\left|\nabla u_{t}(t)\right|^{\beta} d x \\
& -a \int_{\Omega}\left|u_{t}(t)\right|^{m} d x-\int_{\Gamma_{1}} h(x) f(x) y_{t}^{2}(t) d \Gamma \leq 0
\end{aligned}
$$

for any regular solution. Hence we get $E(t) \leq E(0) \forall t \geq 0$. 
By setting $H(t)=-E(t)$, we deduce

$$
0<H(0) \leq H(t) \leq \frac{b}{p} \int_{\Omega}|u(t)|^{p} d x, \quad \forall \geq 0
$$

Now, we define

$$
L(t)=H^{1-\sigma}(t)+\varepsilon \int_{\Omega} u(t) u_{t}(t) d x-\frac{\varepsilon}{2} \int_{\Gamma_{1}} h(x) f(x) y^{2}(t) d \Gamma-\varepsilon \int_{\Gamma_{1}} h(x) u(t) y(t) d \Gamma
$$

for $\varepsilon$ small to be chosen later and

$$
0<\sigma \leq \min \left\{\frac{\alpha-2}{p}, \frac{\alpha-\beta}{p(\beta-1)}, \frac{p-m}{p(m-1)}, \frac{\alpha-2}{2 \alpha}\right\}
$$

Our goal is to show that $L(t)$ satisfies a differential inequality of the form

$$
L^{\prime}(t) \geq \xi L^{q}(t), \quad q>1
$$

This, of course, will lead to a blow-up in finite time.

By taking a derivative of (2.5), we get

$$
\begin{aligned}
L^{\prime}(t)= & (1-\sigma) H^{-\sigma}(t) H^{\prime}(t)+\varepsilon \int_{\Omega} u_{t}^{2}(t) d x+\varepsilon \int_{\Omega} u(t) u_{t t}(t) d x \\
& -\varepsilon \int_{\Gamma_{1}} h(x) f(x) y(t) y_{t}(t) d \Gamma-\varepsilon \int_{\Gamma_{1}} h(x) u_{t}(t) y(t) d \Gamma \\
& -\varepsilon \int_{\Gamma_{1}} h(x) u(t) y_{t}(t) d \Gamma .
\end{aligned}
$$

By using Eqs. (1.1)-(1.4), estimate (2.8) becomes

$$
\begin{aligned}
L^{\prime}(t)= & (1-\sigma) H^{-\sigma}(t) H^{\prime}(t)+\varepsilon \int_{\Omega} u_{t}^{2}(t) d x \\
& +\varepsilon \int_{\Omega} u(t)\left[\Delta u_{t}(t)+\operatorname{div}\left(|\nabla u(t)|^{\alpha-2} \nabla u(t)\right)+\operatorname{div}\left(\left|\nabla u_{t}(t)\right|^{\beta-2} \nabla u_{t}(t)\right)\right. \\
& \left.-a\left|u_{t}(t)\right|^{m-2} u_{t}(t)+b|u(t)|^{p-2} u(t)\right] d x-\varepsilon \int_{\Gamma_{1}} h(x) f(x) y(t) y_{t}(t) d \Gamma \\
& -\varepsilon \int_{\Gamma_{1}} h(x) u_{t}(t) y(t) d \Gamma-\varepsilon \int_{\Gamma_{1}} h(x) u(t) y_{t}(t) d \Gamma \\
= & (1-\sigma) H^{-\sigma}(t) H^{\prime}(t)+\varepsilon \int_{\Omega} u_{t}^{2}(t) d x-\varepsilon \int_{\Omega} \nabla u_{t}(t) \nabla u(t) d x \\
& -\varepsilon \int_{\Omega}|\nabla u(t)|^{\alpha} d x-\varepsilon \int_{\Omega}\left(\left|\nabla u_{t}(t)\right|^{\beta-2} \nabla u_{t}(t)\right) \nabla u(t) d x \\
& -a \varepsilon \int_{\Omega}\left|u_{t}(t)\right|^{m-2} u_{t}(t) u(t) d x+b \varepsilon \int_{\Omega}|u(t)|^{p} d x \\
& +\varepsilon \int_{\Gamma_{1}}\left(\frac{\partial u_{t}(t)}{\partial v}+|\nabla u(t)|^{\alpha-2} \frac{\partial u(t)}{\partial v}+\left|\nabla u_{t}(t)\right|^{\beta-2} \frac{\partial u_{t}(t)}{\partial v}\right) u(t) d \Gamma \\
& -\varepsilon \int_{\Gamma_{1}} h(x) f(x) y(t) y_{t}(t) d \Gamma-\varepsilon \int_{\Gamma_{1}} h(x) u_{t}(t) y(t) d \Gamma-\varepsilon \int_{\Gamma_{1}} h(x) u(t) y_{t}(t) d \Gamma
\end{aligned}
$$




$$
\begin{aligned}
= & (1-\sigma) H^{-\sigma}(t) H^{\prime}(t)+\varepsilon \int_{\Omega} u_{t}^{2}(t) d x-\varepsilon \int_{\Omega} \nabla u_{t}(t) \nabla u(t) d x \\
& -\varepsilon \int_{\Omega}|\nabla u(t)|^{\alpha} d x-\varepsilon \int_{\Omega}\left(\left|\nabla u_{t}(t)\right|^{\beta-2} \nabla u_{t}(t)\right) \nabla u(t) d x \\
& -a \varepsilon \int_{\Omega}\left|u_{t}(t)\right|^{m-2} u_{t}(t) u(t) d x+b \varepsilon \int_{\Omega}|u(t)|^{p} d x+\varepsilon \int_{\Gamma_{1}} h(x) q(x) y^{2}(t) d \Gamma .
\end{aligned}
$$

Exploiting Hölder's and Young's inequalities, for any $\eta, \mu, \delta>0$, we obtain

$$
\begin{aligned}
& \int_{\Omega}\left|u_{t}(t)\right|^{m-2} u_{t}(t) u(t) d x \leq \frac{\eta^{m}}{m} \int_{\Omega}|u(t)|^{m} d x+\frac{m-1}{m} \eta^{-\frac{m}{m-1}} \int_{\Omega}\left|u_{t}(t)\right|^{m} d x, \\
& \int_{\Omega} \nabla u_{t}(t) \nabla u(t) d x \leq \frac{1}{4 \mu} \int_{\Omega}|\nabla u(t)|^{2} d x+\mu \int_{\Omega}\left|\nabla u_{t}(t)\right|^{2} d x, \\
& \int_{\Omega}\left|\nabla u_{t}(t)\right|^{\beta-2} \nabla u_{t}(t) \nabla u(t) d x \leq \frac{\delta^{\beta}}{\beta} \int_{\Omega}|\nabla u(t)|^{\beta} d x+\frac{\beta-1}{\beta} \delta^{-\frac{\beta}{\beta-1}} \int_{\Omega}\left|\nabla u_{t}(t)\right|^{\beta} d x .
\end{aligned}
$$

A substitution of (2.10)-(2.12) in (2.9) yields

$$
\begin{aligned}
L^{\prime}(t) \geq & (1-\sigma) H^{-\sigma}(t) H^{\prime}(t)+\varepsilon \int_{\Omega} u_{t}^{2}(t) d x-\frac{\varepsilon}{4 \mu} \int_{\Omega}|\nabla u(t)|^{2} d x \\
& -\varepsilon \mu \int_{\Omega}\left|\nabla u_{t}(t)\right|^{2} d x-\varepsilon \int_{\Omega}|\nabla u(t)|^{\alpha} d x-\frac{\varepsilon \delta^{\beta}}{\beta} \int_{\Omega}|\nabla u(t)|^{\beta} d x \\
& -\frac{\varepsilon(\beta-1)}{\beta} \delta^{-\frac{\beta}{\beta-1}} \int_{\Omega}\left|\nabla u_{t}(t)\right|^{\beta} d x-\frac{a \varepsilon \eta^{m}}{m} \int_{\Omega}|u(t)|^{m} d x \\
& -\frac{a \varepsilon(m-1)}{m} \eta^{-\frac{m}{m-1}} \int_{\Omega}\left|u_{t}(t)\right|^{m} d x+b \varepsilon \int_{\Omega}|u(t)|^{p} d x \\
& +\varepsilon \int_{\Gamma_{1}} h(x) q(x) y^{2}(t) d \Gamma .
\end{aligned}
$$

Therefore, by choosing $\eta, \mu, \delta$ so that

$$
\begin{aligned}
& \eta^{-\frac{m}{m-1}}=M_{1} H^{-\sigma}(t), \\
& \mu=M_{2} H^{-\sigma}(t), \\
& \delta^{-\frac{\beta}{\beta-1}}=M_{3} H^{-\sigma}(t)
\end{aligned}
$$

for $M_{1}, M_{2}, M_{3}$ to be specified later, and using (2.13), we arrive at

$$
\begin{aligned}
L^{\prime}(t) \geq & (1-\sigma) H^{-\sigma}(t) H^{\prime}(t)+\varepsilon \int_{\Omega} u_{t}^{2}(t) d x-\frac{\varepsilon}{4 M_{2}} H^{\sigma}(t) \int_{\Omega}|\nabla u(t)|^{2} d x \\
& -\varepsilon \int_{\Omega}|\nabla u(t)|^{\alpha} d x-\frac{\varepsilon M_{3}^{-(\beta-1)}}{\beta} H^{\sigma(\beta-1)}(t) \int_{\Omega}|\nabla u(t)|^{\beta} d x \\
& -\frac{a \varepsilon}{m} M_{1}^{-(m-1)} H^{\sigma(m-1)}(t) \int_{\Omega}|u(t)|^{m} d x+b \varepsilon \int_{\Omega}|u(t)|^{p} d x \\
& -\varepsilon\left[M_{2} \int_{\Omega}\left|\nabla u_{t}(t)\right|^{2} d x+\frac{\beta-1}{\beta} M_{3} \int_{\Omega}\left|\nabla u_{t}(t)\right|^{\beta} d x\right. \\
& \left.+\frac{a(m-1)}{m} M_{1} \int_{\Omega}\left|u_{t}(t)\right|^{m} d x\right] H^{-\sigma}(t)+\varepsilon \int_{\Gamma_{1}} h(x) q(x) y^{2}(t) d \Gamma .
\end{aligned}
$$


If $M=M_{2}+\frac{(\beta-1) M_{3}}{\beta}+\frac{(m-1) M_{1}}{m}$, then (2.14) takes the form

$$
\begin{aligned}
L^{\prime}(t) \geq & (1-\sigma-\varepsilon M) H^{-\sigma}(t) H^{\prime}(t)+\varepsilon \int_{\Omega} u_{t}^{2}(t) d x-\frac{\varepsilon}{4 M_{2}} H^{\sigma}(t) \int_{\Omega}|\nabla u(t)|^{2} d x \\
& -\varepsilon \int_{\Omega}|\nabla u(t)|^{\alpha} d x-\frac{\varepsilon M_{3}^{-(\beta-1)}}{\beta} H^{\sigma(\beta-1)}(t) \int_{\Omega}|\nabla u(t)|^{\beta} d x \\
& -\frac{a \varepsilon}{m} M_{1}^{-(m-1)} H^{\sigma(m-1)}(t) \int_{\Omega}|u(t)|^{m} d x+b \varepsilon \int_{\Omega}|u(t)|^{p} d x \\
& +\varepsilon M H^{-\sigma}(t) \int_{\Gamma_{1}} h(x) f(x) y_{t}^{2}(t) d \Gamma+\varepsilon \int_{\Gamma_{1}} h(x) q(x) y^{2}(t) d \Gamma .
\end{aligned}
$$

Then we use the embedding $L^{p}(\Omega) \hookrightarrow L^{m}(\Omega)$ and (2.4) to get

$$
H^{\sigma(m-1)}(t) \int_{\Omega}|u(t)|^{m} d x \leq\left(\frac{b}{p}\right)^{\sigma(m-1)}\left(\int_{\Omega}|u(t)|^{p} d x\right)^{\frac{m+\sigma p(m-1)}{p}} .
$$

We also exploit the inequality

$$
\int_{\Omega}|\nabla u(t)|^{2} d x \leq c\left(\int_{\Omega}|\nabla u(t)|^{\alpha} d x\right)^{\frac{2}{\alpha}}
$$

the embedding $W^{1, \alpha}(\Omega) \hookrightarrow H^{1}(\Omega)$ and (2.4) to obtain

$$
H^{\sigma}(t) \int_{\Omega}|\nabla u(t)|^{2} d x \leq c\left(\frac{b}{p}\right)^{\sigma}\left(\int_{\Omega}|\nabla u(t)|^{\alpha} d x\right)^{\frac{p \sigma+2}{\alpha}} .
$$

Since $\alpha>\beta$, we obtain

$$
\int_{\Omega}|\nabla u(t)|^{\beta} d x \leq c\left(\int_{\Omega}|\nabla u(t)|^{\alpha} d x\right)^{\frac{\beta}{\alpha}}
$$

we derive

$$
H^{\sigma(\beta-1)}(t) \int_{\Omega}|\nabla u(t)|^{\beta} d x \leq c\left(\frac{b}{p}\right)^{\sigma(\beta-1)}\left(\int_{\Omega}|\nabla u(t)|^{\alpha} d x\right)^{\frac{p \sigma(\beta-1)+\beta}{\alpha}}
$$

where $c$ is a constant depending on $\Omega$ only. By using (2.6) and the inequality

$$
z^{v} \leq z+1 \leq\left(1+\frac{1}{a}\right)(z+a), \quad \forall z \geq 0,0<v<1, a \geq 0
$$

we get the following inequalities:

$$
\begin{aligned}
\left(\int_{\Omega}|u(t)|^{p} d x\right)^{\frac{m+\sigma p(m-1)}{p}} & \leq c\left(\int_{\Omega}|\nabla u(t)|^{\alpha} d x\right)^{\frac{m+\sigma p(m-1)}{\alpha}} \\
& \leq d\left(\int_{\Omega}|\nabla u(t)|^{\alpha} d x+H(0)\right) \\
& \leq d\left(\int_{\Omega}|\nabla u(t)|^{\alpha} d x+H(t)\right), \quad \forall t \geq 0,
\end{aligned}
$$




$$
\left(\int_{\Omega}|\nabla u(t)|^{\alpha} d x\right)^{\frac{p \sigma+2}{\alpha}} \leq d\left(\int_{\Omega}|\nabla u(t)|^{\alpha} d x+H(t)\right), \quad \forall t \geq 0,
$$

and

$$
\left(\int_{\Omega}|\nabla u(t)|^{\alpha} d x\right)^{\frac{p \sigma(\beta-1)+\beta}{\alpha}} \leq d\left(\int_{\Omega}|\nabla u(t)|^{\alpha} d x+H(t)\right), \quad \forall t \geq 0
$$

where $d=1+1 / H(0), a=H(0)$. Inserting (2.16)-(2.18) and (2.20)-(2.22) into (2.15), we deduce

$$
\begin{aligned}
L^{\prime}(t) \geq & (1-\sigma-\varepsilon M) H^{-\sigma}(t) H^{\prime}(t) \\
& +k H(t)+\left(\varepsilon+\frac{k}{2}\right) \int_{\Omega} u_{t}^{2}(t) d x \\
& -\frac{\varepsilon c_{2}}{M_{2}}\left(\int_{\Omega}|\nabla u(t)|^{\alpha} d x+H(t)\right)-\varepsilon \int_{\Omega}|\nabla u(t)|^{\alpha} d x \\
& -\frac{\varepsilon c_{3}}{M_{3}^{\beta-1}}\left(\int_{\Omega}|\nabla u(t)|^{\alpha} d x+H(t)\right)+\frac{k}{\alpha} \int_{\Omega}|\nabla u(t)|^{\alpha} d x \\
& -\frac{\varepsilon c_{1}}{M_{1}^{m-1}}\left(\int_{\Omega}|\nabla u(t)|^{\alpha} d x+H(t)\right)+b\left(\varepsilon-\frac{k}{p}\right) \int_{\Omega}|u(t)|^{p} d x \\
& +\varepsilon M H^{-\sigma}(t) \int_{\Gamma_{1}} h(x) f(x) y_{t}^{2}(t) d \Gamma+\left(\varepsilon+\frac{k}{2}\right) \int_{\Gamma_{1}} h(x) q(x) y^{2}(t) d \Gamma
\end{aligned}
$$

for some constant $k$ and $c_{1}=\frac{a c d}{m}\left(\frac{b}{p}\right)^{\sigma(m-1)}, c_{2}=\frac{c d}{4}\left(\frac{b}{p}\right)^{\sigma}, c_{3}=\frac{c d}{\beta}\left(\frac{b}{p}\right)^{\sigma(\beta-1)}$.

Using $k=\varepsilon p$, we arrive at

$$
\begin{aligned}
L^{\prime}(t) \geq & (1-\sigma-\varepsilon M) H^{-\sigma}(t) H^{\prime}(t)+\varepsilon\left(\frac{p+2}{2}\right) \int_{\Omega} u_{t}^{2}(t) d x \\
& +\varepsilon\left(p-\frac{c_{2}}{M_{2}}-\frac{c_{3}}{M_{3}^{\beta-1}}-\frac{c_{1}}{M_{1}^{m-1}}\right) H(t) \\
& +\varepsilon\left(\frac{p}{\alpha}-\frac{c_{2}}{M_{2}}-\frac{c_{3}}{M_{3}^{\beta-1}}-\frac{c_{1}}{M_{1}^{m-1}}-1\right) \int_{\Omega}|\nabla u(t)|^{\alpha} d x \\
& +\varepsilon M H^{-\sigma}(t) \int_{\Gamma_{1}} h(x) f(x) y_{t}^{2}(t) d \Gamma+\varepsilon\left(\frac{p+2}{2}\right) \int_{\Gamma_{1}} h(x) q(x) y^{2}(t) d \Gamma .
\end{aligned}
$$

At this point, by choosing $M_{1}, M_{2}, M_{3}$ large enough and using

$$
\varepsilon M H^{-\sigma}(t) \int_{\Gamma_{1}} h(x) f(x) y_{t}^{2}(t) d \Gamma>0
$$

we have

$$
\begin{aligned}
L^{\prime}(t) \geq & (1-\sigma-\varepsilon M) H^{-\sigma}(t) H^{\prime}(t) \\
& +r \varepsilon\left(H(t)+\int_{\Omega} u_{t}^{2}(t) d x+\int_{\Omega}|\nabla u(t)|^{\alpha} d x+\int_{\Gamma_{1}} h(x) q(x) y^{2}(t) d \Gamma\right),
\end{aligned}
$$

where $r$ is a positive constant (this is possible since $p>\alpha$ ). 
We choose $0<\varepsilon<\frac{1-\sigma}{M}$ so that

$$
L(0)=H^{1-\sigma}(0)+\varepsilon \int_{\Omega} u_{0} u_{1} d x-\frac{\varepsilon}{2} \int_{\Gamma_{1}} h(x) f(x) y_{0}^{2} d \Gamma-\varepsilon \int_{\Gamma_{1}} h(x) u_{0} y_{0} d \Gamma>0
$$

Then from (2.23) we get

$$
L(t) \geq L(0)>0, \quad \forall t \geq 0,
$$

and

$$
L^{\prime}(t) \geq r \varepsilon\left(H(t)+\int_{\Omega} u_{t}^{2}(t) d x+\int_{\Omega}|\nabla u(t)|^{\alpha} d x+\int_{\Gamma_{1}} h(x) q(x) y^{2}(t) d \Gamma\right) .
$$

On the other hand, from (2.5) and $f, h>0$, we have

$$
L(t) \leq H^{1-\sigma}(t)+\varepsilon \int_{\Omega} u(t) u_{t}(t) d x-\varepsilon \int_{\Gamma_{1}} h(x) u(t) y(t) d \Gamma .
$$

Consequently, the above estimate leads to

$$
L^{\frac{1}{1-\sigma}}(t) \leq C(\varepsilon, \sigma)\left[H(t)+\left(\int_{\Omega} u(t) u_{t}(t) d x\right)^{\frac{1}{1-\sigma}}+\left(\int_{\Gamma_{1}} h(x) u(t) y(t) d \Gamma\right)^{\frac{1}{1-\sigma}}\right] .
$$

From Hölder's inequality, we obtain

$$
\begin{aligned}
\int_{\Omega} u(t) u_{t}(t) d x & \leq\left(\int_{\Omega} u_{t}^{2}(t) d x\right)^{\frac{1}{2}}\left(\int_{\Omega} u^{2}(t) d x\right)^{\frac{1}{2}} \\
& \leq c\left(\int_{\Omega} u_{t}^{2}(t) d x\right)^{\frac{1}{2}}\left(\int_{\Omega}|u(t)|^{\alpha} d x\right)^{\frac{1}{\alpha}},
\end{aligned}
$$

where $c$ is the positive constant which comes from the embedding $L^{\alpha}(\Omega) \hookrightarrow L^{2}(\Omega)$. This inequality implies that there exists a positive constant $c_{4}>0$ such that

$$
\left(\int_{\Omega} u(t) u_{t}(t) d x\right)^{\frac{1}{1-\sigma}} \leq c_{4}\left(\int_{\Omega}|u(t)|^{\alpha} d x\right)^{\frac{1}{(1-\sigma) \alpha}}\left(\int_{\Omega} u_{t}^{2}(t) d x\right)^{\frac{1}{2(1-\sigma)}} .
$$

Applying Young's inequality to the right-hand side of the preceding inequality, we have a positive constant, also denoted by $c>0$, such that

$$
\left(\int_{\Omega} u(t) u_{t}(t) d x\right)^{\frac{1}{1-\sigma}} \leq c\left[\left(\int_{\Omega}|u(t)|^{\alpha} d x\right)^{\frac{\mu}{(1-\sigma) \alpha}}+\left(\int_{\Omega} u_{t}^{2}(t) d x\right)^{\frac{\theta}{2(1-\sigma)}}\right]
$$

for $\frac{1}{\mu}+\frac{1}{\theta}=1$. We take $\theta=2(1-\sigma)$, hence $\mu=2(1-\sigma) /(1-2 \sigma)$, to get

$$
\left(\int_{\Omega} u(t) u_{t}(t) d x\right)^{\frac{1}{1-\sigma}} \leq c\left[\left(\int_{\Omega}|u(t)|^{\alpha} d x\right)^{\frac{2}{(1-2 \sigma) \alpha}}+\int_{\Omega} u_{t}^{2}(t) d x\right] .
$$


By Poincare's inequality, we obtain

$$
\left(\int_{\Omega} u(t) u_{t}(t) d x\right)^{\frac{1}{1-\sigma}} \leq c\left[\left(\int_{\Omega}|\nabla u(t)|^{\alpha} d x\right)^{\frac{2}{(1-2 \sigma) \alpha}}+\int_{\Omega} u_{t}^{2}(t) d x\right] .
$$

We use (2.6) and the algebraic inequality (2.19) with $z=\|\nabla u(t)\|_{\alpha}^{\alpha}, d=1+1 / H(0), a=H(0)$, $v=2 / \alpha(1-2 \sigma)$, condition (2.6) on $\sigma$ ensures that $0<\nu<1$, and it follows that

$$
z^{v} \leq d(z+H(0)) \leq d(z+H(t))
$$

Therefore, from (2.20), there exists a positive constant, denoted by $c_{4}$, such that for all $t \geq 0$,

$$
\left(\int_{\Omega} u(t) u_{t}(t) d x\right)^{\frac{1}{1-\sigma}} \leq c_{4}\left[H(t)+\|\nabla u(t)\|_{\alpha}^{\alpha}+\left\|u_{t}(t)\right\|_{2}^{2}\right] .
$$

Furthermore, by the same method, we have

$$
\begin{aligned}
\int_{\Gamma_{1}} h(x) u(t) y(t) d \Gamma & =\left|\int_{\Gamma_{1}} \frac{h(x) q(x)}{q(x)} u(t) y(t) d \Gamma\right| \\
& \leq \frac{\|h\|_{\infty}^{\frac{1}{2}}\|q\|_{\infty}^{\frac{1}{2}}}{q_{0}}\left(\int_{\Gamma_{1}} h(x) q(x) y^{2}(t) d \Gamma\right)^{\frac{1}{2}}\left(\int_{\Gamma_{1}} u^{2}(t) d \Gamma\right)^{\frac{1}{2}} .
\end{aligned}
$$

Using the embedding $W_{0}^{1, \alpha}(\Omega) \hookrightarrow L^{2}\left(\Gamma_{1}\right)$ and Hölder's inequality, we get

$$
\int_{\Gamma_{1}} h(x) u(t) y(t) d \Gamma \leq c_{5} \frac{\|h\|_{\infty}^{\frac{1}{2}}\|q\|_{\infty}^{\frac{1}{2}}}{q_{0}}\left(\int_{\Gamma_{1}} h(x) q(x) y^{2}(t) d \Gamma\right)^{\frac{1}{2}}\left(\int_{\Omega}|\nabla u(t)|^{\alpha} d x\right)^{\frac{1}{\alpha}} .
$$

Consequently, there exists a positive constant $c_{5}=c_{5}\left(\|h\|_{\infty},\|q\|_{\infty}, q_{0}, \sigma, \alpha\right)$ such that

$$
\left(\int_{\Gamma_{1}} h(x) u(t) y(t) d \Gamma\right)^{\frac{1}{1-\sigma}} \leq c_{5}\left(\int_{\Gamma_{1}} h(x) q(x) y^{2}(t) d \Gamma\right)^{\frac{1}{2(1-\sigma)}}\left(\int_{\Omega}|\nabla u(t)|^{\alpha} d x\right)^{\frac{1}{\alpha(1-\sigma)}} .
$$

Using Young's inequality exactly as in (2.26), we write

$$
\left(\int_{\Gamma_{1}} h(x) u(t) y(t) d \Gamma\right)^{\frac{1}{1-\sigma}} \leq c_{6}\left[\int_{\Gamma_{1}} h(x) q(x) y^{2}(t) d \Gamma+\left(\int_{\Omega}|\nabla u(t)|^{\alpha} d x\right)^{\frac{2}{\alpha(1-2 \sigma)}}\right],
$$

where $c_{6}$ is a positive constant depending on $c_{5}$ and $\alpha$. Consequently, applying once again the algebraic inequality (2.19) with $z=\|\nabla u(t)\|_{\alpha}^{\alpha}, v=2 / \alpha(1-2 \sigma)$ and making use of (2.6), we obtain by the same method as above

$$
\left(\int_{\Gamma_{1}} h(x) u(t) y(t) d \Gamma\right)^{\frac{1}{1-\sigma}} \leq c_{7}\left[H(t)+\|\nabla u(t)\|_{\alpha}^{\alpha}+\int_{\Gamma_{1}} h(x) q(x) y^{2}(t) d \Gamma\right],
$$

where $c_{7}$ is a positive constant. From (2.25), (2.26) and (2.27), we arrive at

$$
L^{\frac{1}{1-\sigma}}(t) \leq c\left[H(t)+\|\nabla u(t)\|_{\alpha}^{\alpha}+\left\|u_{t}(t)\right\|_{2}^{2}+\int_{\Gamma_{1}} h(x) q(x) y^{2}(t) d \Gamma\right]
$$


where $c$ is a positive constant. Consequently, a combination of (2.24) and (2.28), for some $\xi>0$, yields

$$
L^{\prime}(t) \geq \xi L^{\frac{1}{1-\sigma}}(t), \quad \forall t \geq 0 .
$$

Integration of (2.29) over $(0, t)$ gives

$$
L^{\frac{\sigma}{1-\sigma}}(t) \geq \frac{1}{L^{\frac{-\sigma}{1-\sigma}}(0)-\frac{\xi \sigma}{1-\sigma} t}, \quad \forall t \geq 0 .
$$

Hence $L(t)$ blows up in finite time

$$
T^{*} \leq \frac{1-\sigma}{\xi \sigma L^{\frac{\sigma}{1-\sigma}}(0)}
$$

Thus the proof of Theorem 2.1 is complete.

\section{Competing interests}

The authors declare that they have no competing interests.

Authors' contributions

All authors contributed equally to the writing of this paper. All authors read and approved the final manuscript.

\section{Author details}

'Division of Mathematical Sciences, Pukyong National University, Busan, 608-737, South Korea. ${ }^{2}$ Department of Mathematics, Pusan National University, Busan, 609-735, South Korea. ${ }^{3}$ Institute of Liberal Education, Catholic University of Daegu, Gyeongsan, 712-702, South Korea.

\section{Acknowledgements}

This research was supported by Basic Science Research Program through the National Research Foundation of Korea (NRF) funded by the Ministry of Education (NRF-2016R1A1B03930361).

Received: 7 December 2016 Accepted: 13 February 2017 Published online: 28 March 2017

\section{References}

1. Morse, PM, Ingard, KU: Theoretical Acoustics. McGraw-Hill, New York (1968)

2. Beale, JT, Rosencrans, SI: Acoustic boundary conditions. Bull. Am. Math. Soc. 80, 1276-1278 (1974)

3. Boukhatem, Y, Benabderrahmane, B: Polynomial decay and blow up of solutions for variable coefficients viscoelastic wave equation with acoustic boundary conditions. Acta Math. Sin. Engl. Ser. 32(2), 153-174 (2016)

4. Boukhatem, Y, Benabderrahmane, B: Existence and decay of solutions for a viscoelastic wave equation with acoustic boundary conditions. Nonlinear Anal. 97, 191-209 (2014)

5. Graber, PJ, Said-Houari, B: On the wave equation with semilinear porous acoustic boundary conditions. J. Differ. Equ. 252, 4898-4941 (2012)

6. Wu, ST: Non-existence of global solutions for a class of wave equations with nonlinear damping and source terms. Proc. R. Soc. Edinb. A 141(4), 865-880 (2011)

7. Messaoudi, SA: Blow-up of positive-initial-energy solutions of a nonlinear viscoelastic hyperbolic equation. J. Math. Anal. Appl. 320, 902-915 (2006)

8. Messaoudi, SA: Blow up in a non-linearly damping wave equation. Math. Nachr. 231, 1-7 (2001)

9. Messaoudi, SI, Houari, BS: Global non-existence of solutions of a class of wave equations with non-linear damping and source terms. Math. Methods Appl. Sci. 27, 1687-1696 (2004)

10. Ball, J: Remarks on blow-up and non-existence theorems for non-linear evolutions equations. Quarterly J. Math. Oxford Series 28(2), 473-486 (1977)

11. Hao, J, Zhang, Y, Li, S: Global existence and blow-up phenomena for a nonlinear wave equation. Nonlinear Anal. 71, 4823-4832 (2009)

12. Levine, HA: Some additional remarks on the non-existence of global solutions to non-linear wave equation. SIAM J. Math. Anal. 5, 138-146 (1974)

13. Yang, Z: Blow up of solutions for a class of non-linear evolution equations with non-linear damping and source terms. Math. Methods Appl. Sci. 25, 825-833 (2002)

14. Piskin, E: On the decay and blow up of solutions for a quasilinear hyperbolic equations with nonlinear damping and source terms. Bound. Value Probl. 2015(127), 1 (2015)

15. Ang, DD, Dinh, APN: Strong solutions of quasilinear wave equation with non-linear damping. SIAM J. Math. Anal. 19 337-347 (1988)

16. Kalantarov, VK, Ladyzhenskaya, OA: The occurrence of collapse for quasilinear equations of parabolic and hyperbolic type. J. Sov. Math. 10, 53-70 (1978) 
17. Levine, HA, Serrin, J: A global non-existence theorem for quasilinear evolution equation with dissipation. Archives for Rational Mechanics and Analysis 137, 341-361 (1997)

18. Levine, HA, Park, SR: Global existence and global non-existence of solutions of the Cauchy problem for a non-linearly damped wave equation. J. Math. Anal. Appl. 228, 181-205 (1998)

Submit your manuscript to a SpringerOpen ${ }^{\circ}$ journal and benefit from:

- Convenient online submission

Rigorous peer review

- Immediate publication on acceptance

- Open access: articles freely available online

- High visibility within the field

- Retaining the copyright to your article

Submit your next manuscript at $>$ springeropen.com 